\title{
Improving polymer solar cell performances by manipulating the self- organization of polymer
}

\author{
Feng-xian Xie, ${ }_{3}^{1}$ Wallace C. H. Choy, ${ }^{1, a)}$ Xiaolong Zhu, ${ }^{1}$ Xiaolong $\mathrm{Li}^{2},{ }^{2}$ Zhong $\mathrm{Li}^{2},{ }^{2}$ and \\ Chunjun Liang ${ }^{3}$ \\ ${ }^{1}$ Department of Electrical and Electronic Engineering, The University of Hong Kong, Pokfulam Road, \\ Hong Kong \\ ${ }^{2}$ Shanghai Synchrotron Radiation Facility, Shanghai Institute of Applied Physics, Chinese Academy of \\ Sciences, Shanghai 201204, People's Republic of China \\ ${ }^{3}$ Institute of Optoelectronical Technology, School of Science, Beijing JiaoTong University, Beijing 100044, \\ People's Republic of China
}

(Received 8 April 2011; accepted 17 May 2011; published online 13 June 2011)

\begin{abstract}
We have investigated driving force effects on the ordering of polymer, which is a key factor of self-assembly of soft materials. By turning the substrate up-side-down, the downward driving force can form in solution film-growth process and affect the self-organization of polymer chains and domains. We introduce Brown's capillarity theory [J. Polym. Sci., Polym. Phys. Ed. 22, 423 (1956)] to describe the film formation. Our results show that the better chain and lamellae packing of polymer make hole transport, carrier balance, and power conversion efficiency of annealed and unannealed devices improve even with thick active-layers as compared to conventional devices. (C) 2011 American Institute of Physics. [doi:10.1063/1.3599488]
\end{abstract}

Bulk-heterojunction polymer solar cells (PSCs) have attracted considerable attention for its remarkable advantages. ${ }^{1,2}$ Meanwhile, the solution based film-growth dynamics (the slow growth) of polymer blends has become one of the crucial processes which modifies the polymer morphology to improve the efficiency from bulkheterojunction structures. ${ }^{3,4}$ The slow growth will assist the formation of self-organized ordered structures in poly(3hexylthiophene) (P3HT):[6,6]-phenyl C61-butyric acid methyl ester (PCBM) blend system. The electrical and optical properties of polymer blends will also be modified by solution parameters and spin-coating conditions. ${ }^{3,4}$

In this work, we will study driving force effects on the order and phase of crystallization of the blended polymer in solution based self-assembly, which is a key factor of the self-assembly of soft materials ${ }^{5}$ but has been neglected in studying PSC morphology. We investigate driving force effects by flipping the substrate up-side-down (hereafter named as "up-side-down" method) after spin-coating the wet polymer blend.

PSCs with a structure of Indium tin oxide (ITO)/ Poly(3,4-ethylenedioxythiophene poly (styrenesulfonate) (PEDOT:PSS) (Baytron AI4083)/P3HT:PCBM/LiF/Al were fabricated following the method as described elsewhere. ${ }^{6}$ The mixed solution (P3HT: $\mathrm{PCBM}=1: 1,45 \mathrm{mg} / \mathrm{ml}$ ) was spin-coated at $800 \mathrm{rpm}$ and $500 \mathrm{rpm}$ for $45 \mathrm{~s}$, forming films with thicknesses of $210 \mathrm{~nm}$ and $300 \mathrm{~nm}$ respectively. Then the film was quickly transferred to a petri dish for the slow growth for $40 \mathrm{~min}$. The up-side-down film was made by flipping the substrate and letting the wet film face downward during the slow growth. After the slow growth process, the film was annealed at $120{ }^{\circ} \mathrm{C}$ for $10 \mathrm{~min}$ on a hot plate. Then $1 \mathrm{~nm}$ thick of $\mathrm{LiF}$ and $100 \mathrm{~nm} \mathrm{Al}$ were evaporated. Besides fabricating the set of annealed devices stated above, we made

\footnotetext{
a) Author to whom correspondence should be addressed. Electronic mail: chchoy@eee.hku.hk.
}

a set of unannealed devices and films with film thickness 210 $\mathrm{nm}$ for analyzing the effects of forces on the film properties since the annealing afterward will reconstruct polymer morphology. ${ }^{7-9}$

To show and analyze the effects of the driving force on PSCs, we first study unannealed devices. By flipping the substrate to make solution film growth, the short circuit current $\left(J_{s c}\right)$ enhances from $6.56 \mathrm{~mA} / \mathrm{cm}^{2}$ (control) to $7.96 \mathrm{~mA} / \mathrm{cm}^{2}$ (up-side-down) and the fill factor (FF) improves from 0.47 (control) to 0.6 (up-side-down) as shown in Fig. 1(a). As a result, we obtain PCE of $2.64 \%$ for the upside-down device as compared to $1.69 \%$ of the unannealed control device. Meanwhile, from the dark current as shown in Fig. 1(b), we find that the current density of the up-sidedown devices is higher than the conventional one particularly at a forward bias, which indicates a higher charge transport in the up-side-down devices.

To analyze the charge transport, we fabricate unannealed hole-only devices with structures of ITO/PEDOT:PSS/ P3HT:PCBM (up-side-down and conventional)/Au (20 $\mathrm{nm}) / \mathrm{Al}(60 \mathrm{~nm})$ and ITO/Ca (2 nm) / P3HT:PCBM (up-sidedown and conventional)/Au (20 nm)/Al (60 nm) and unannealed electron-only devices of ITO/Ca $(2 \mathrm{~nm}) /$ P3HT:PCBM (up-side-down and conventional)/LiF (1 $\mathrm{nm}) / \mathrm{Al}(60 \mathrm{~nm}){ }^{10}$ As shown in Figs. 1(c) and 1(d), the current density of the up-side-down hole-only device is higher than that of the conventional one. This indicates a better hole transport for the up-side-down device. Meanwhile, the current density of the up-side-down electron-only device is lower than that of the conventional one. Consequently, the increase in hole current density and the decrease in electron current density will make the hole and electron transport more balance as compared to the conventional device for enhancing their performances.

The effects of the up-side-down method on optical properties of polymer blends are illustrated in Fig. 2(a). The absorption spectrum of the up-side-down film shows higher 

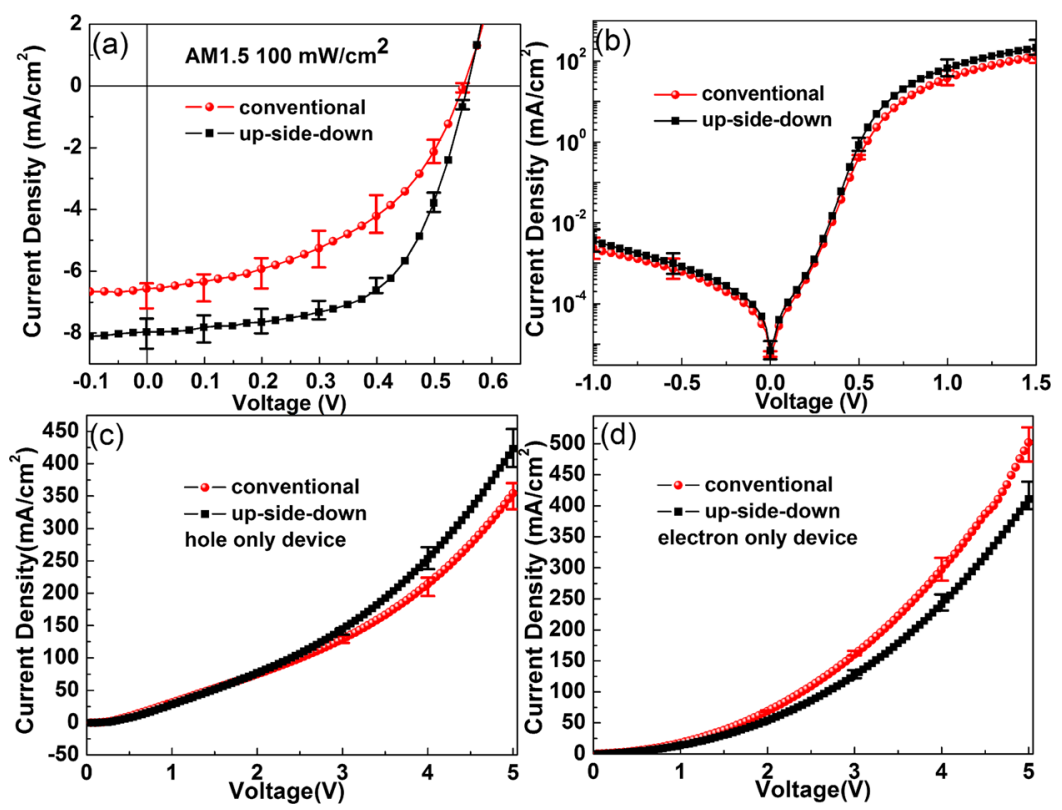

FIG. 1. (Color online) J-V characteristics of conventional and up-side-down nonannealed PSCs: (a) under AM $1.5 \mathrm{G} 100 \mathrm{~mW} / \mathrm{cm}^{2}$ illumination and (b) in the dark. J-V characteristics of (c) hole-only devices and (d) electron-only devices. For each type of PSCs, 12 devices were made and characterized. Their performances were shown as error bars. light absorption and shaper peak at $600 \mathrm{~nm}$ than that of the conventional device due to the improvement of the interchain packing of $\mathrm{P} 3 \mathrm{HT}$ and local order inside the domains. ${ }^{11}$ Incident-photon-to-electron conversion efficiency (IPCE) also improves as the absorption spectrum shown in Fig. 2(b). The maximum IPCE peak of the up-side-down device is $49 \%$ at $\sim 600 \mathrm{~nm}$ while the conventional one is $42 \%$ at $560 \mathrm{~nm}$.

The driving force effects on the morphology formation are studied. According to capillarity theory first proposed by Brown, the polymer film formation from solvent is mainly driven by capillary forces $(\mathrm{Fc})$ which derives from the surface tension of the interstitial solvent. ${ }^{12,13}$ For the conventional film, the driving force of Fc points upward as shown in

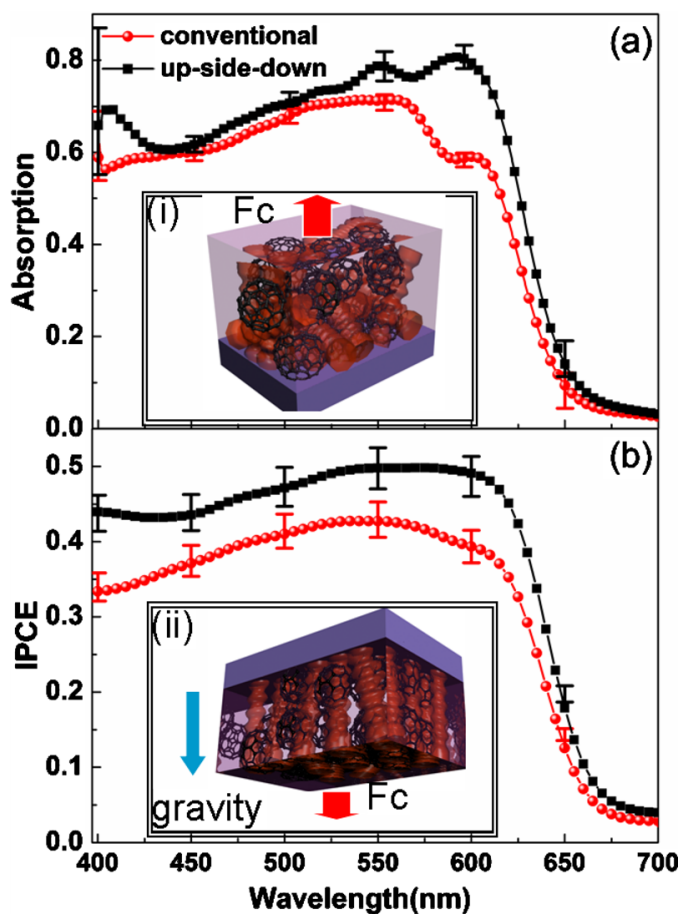

FIG. 2. (Color online) (a) Optical absorption spectra and (b) IPCE of conventional and up-side-down nonannealed devices. Insets (i) and (ii) are schematic diagram of the conventional and up-side-down film, respectively. For each type of PSCs, 12 devices were made and characterized. Their performances were shown as error bars.
Fig. 2(i). The (top) interface between polymer and air is subject to Fc, which makes the polymer start packing from top and crystallize downward. However, in the blend system, the presence of PCBM could also separate out and block the continuous crystallization of P3HT and new nuclei of P3HT therefore form in the blend layer during the solvent evaporation. As a result, the polymer crystallization at different directions happens at various positions, which agrees with atomic force microscopy (AFM) results as discussed later.

For the up-side-down film, the growth process is different from that of the conventional process, which could make the film better ordered. Initially, crystallization is not favor at the interface like the conventional one since the downward solution due to gravity would dissolve the polymer at the surface again. Meanwhile, when the solvent evaporates, nuclei start to crystallize in the wet polymer layer. Until the solvent reaches a critical volume, the capillarity force plays a dominant role in the film formation process. ${ }^{13}$ At that time, the crystals in the polymer layer continuously grow and pack orderly by the downward driving force of $\mathrm{Fc}$, as indicated from AFM results shown in Fig. 3.

The morphology of polymer blends is investigated by AFM as shown in Figs. 3(a) and 3(b). The surface roughness indicates the ordering of polymer self-organization. ${ }^{3}$ For our devices, the conventional film has an rms roughness of 6.94 nm while the up-side-down film has a larger rms roughness of $9.98 \mathrm{~nm}$ implying a higher ordering structure formed in the up-side-down film. The up-side-down film also has wider waviness $(\sim 60 \mathrm{~nm})$ and smoother surface when the conventional one has waviness of $37 \mathrm{~nm}$ and a lot of small humps. The small humps of the conventional film could be due to the disordered packing of polymer crystals grown in different directions as described above. Consequently, the up-sidedown process makes the film has a better polymer ordering and smoother surface which agrees with the previous description of the polymer crystallization.

Figures 3(c) and 3(d) show the two-dimensional (2D) GIXRD patterns and Fig. 3(e) shows the one-dimensional (1D) out-of-plane X-ray profiles of P3HT:PCBM blend measured at Shanghai Synchrotron Radiation Facility. ${ }^{14}$ The intense reflections of (00h) are obtained from 1D out-of-plane 

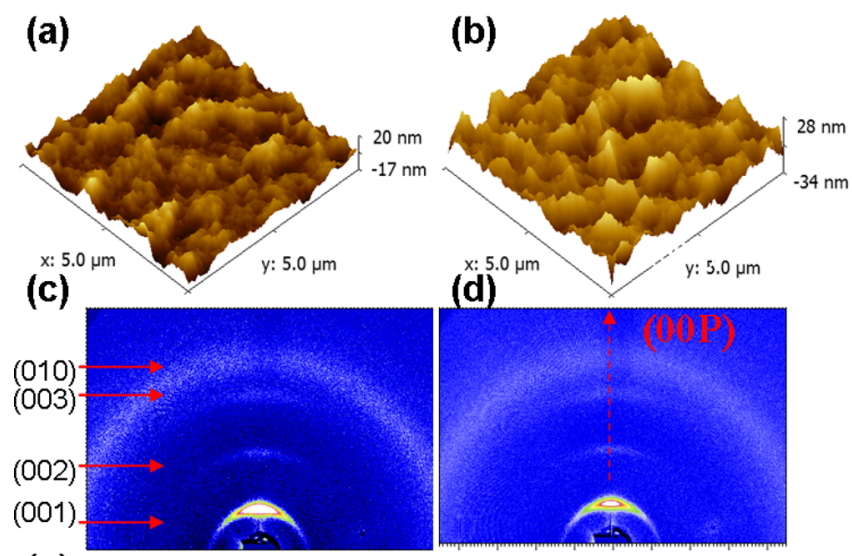

(e)

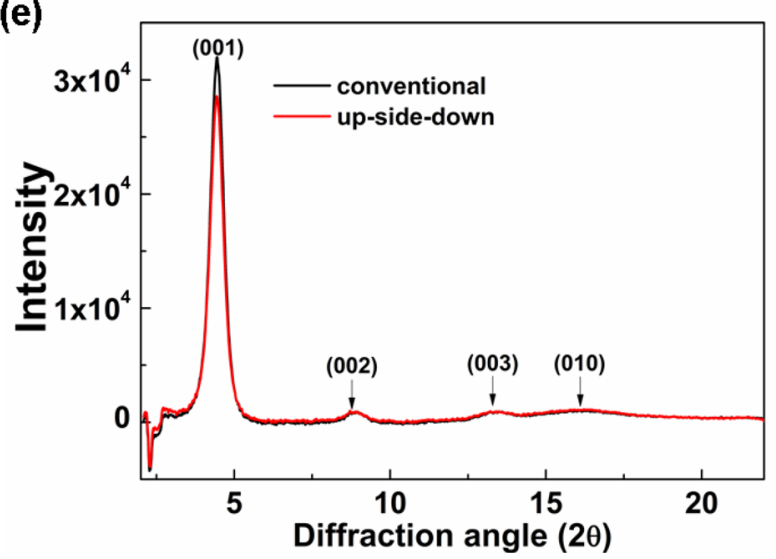

FIG. 3. (Color online) AFM image of (a) conventional film and (b) up-sidedown-film, and 2D GIXRD patterns of (c) conventional film and (d) upside-down film. (e) is the 1D out of plane of GIXRD result at 00P direction; conventional (black) and up-side-down (red) film. All films are nonannealed and prepared by the slow growth method.

$\mathrm{x}$-ray profiles, which indicated a well organized intraplane structure with lamellae oriented normal to the substrate. The peak maximum of the scattering vector $q_{z}$ is at $0.391 \AA^{-1}$ corresponding to an interlayer spacing of about $16.1 \AA$. The film made by the up-side-down method has the same interlayer spacing as that of the conventional method. However, the intensity of the (001) signal of the polymer film made by the up-side-down method is slightly weaker than that of the conventional one, indicating a little smaller size or number of the P3HT crystals formed in up-side-down film. It should be noted that the GIXRD signal is from no more than $10 \mathrm{~nm}$ deep from the polymer/air surface. That means for the conventional film, the amount of crystallization at surface is slightly better than the up-side-down one. From the filmgrowth process discussed above, we find that the better surface crystallization of conventional film is reasonable. While the up-side-down film starts growth process inside the film, the conventional film starts growing from the surface which will favor the crystallization in the region near the polymer/ air surface.

For annealed PSCs, both up-side-down and conventional devices with a typical thickness of about $210 \mathrm{~nm}$ show improved performances by thermal annealing. Our results show that PCE of up-side-down devices is still higher than that of conventional devices, i.e., $3.5 \%$ compared to $3.3 \%$ as shown in Fig. 4. When we increase the active layer to $300 \mathrm{~nm}$, the conventional device performance degrades, due to the in-

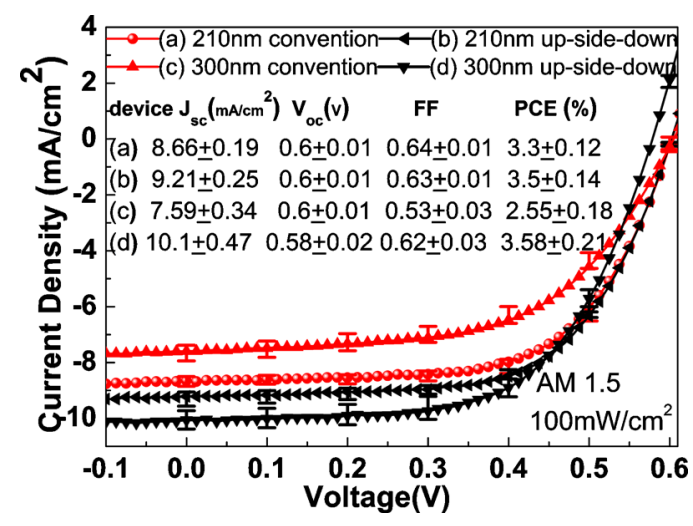

FIG. 4. (Color online) J-V characteristics of annealed PSCs with different thicknesses. Inset shows the performances of PSCs. For each type of PSCs, 12 devices were made and characterized. Their performances were shown as error bars.

crease in recombination of excitons in the thick active layer, $J_{s c}$ reduces to $8.04 \mathrm{~mA} / \mathrm{cm}^{2}$ and PCE reduces to $2.55 \%$. Interestingly, $J_{s c}$ of the up-side-down device is further improved from 9.21 to $10 \mathrm{~mA} / \mathrm{cm}^{2}$ and PCE achieves $3.58 \%$, which can be explained by the better charge transport paths.

In summary, by controlling the driving force in the solution-film growth dynamics through the up-side-down method, the ordering of polymer blends is improved. We introduce Brown's capillarity theory to explain the blended polymer film formation. Our results show that the hole transport, carrier balance, $J_{s c}$, and FF improve, and thus PCE enhances in up-side-down devices due to the better chain packing and local order of polymer domains.

This work is supported by UGC grant (Grant No. 400897) of The University of Hong Kong and the General Research Fund (Grant Nos. HKU712108 and HKU712010) from the Research Grants Council of Hong Kong. We acknowledge the technical support of Xiuhong Li.

${ }^{1}$ G. Dennler, M. C. Scharber, and C. J. Brabec, Adv. Mater. (Weinheim, Ger.) 21, 1323 (2009).

${ }^{2}$ B. C. Thompson and J. M. J. Fréchet, Angew. Chem., Int. Ed. 47, 58 (2008).

${ }^{3}$ G. Li, V. Shrotriya, J. Huang, Y. Yao, T. Moriarty, K. Emery, and Y. Yang, Nature Mater. 4, 864 (2005).

${ }^{4}$ Y. Yao, J. Hou, Z. Xu, G. Li, and Y. Yang, Adv. Funct. Mater. 18, 1783 (2008).

${ }^{5}$ C. J. Morris, S. A. Stauth, and B. A. Parviz, IEEE Trans. Adv. Packag. 28 600 (2005).

${ }^{6}$ C. D. Wang and W. C. H. Choy, Sol. Energy Mater. Sol. Cells 95, 904 (2011).

${ }^{7}$ M. Campoy-Quiles, T. Ferenczi, T. Agostinelli, P. G. Etchegoin, Y. Kim, T. D. Anthopoulos, P. N. Stavrinou, D. D. C. Bradley, and J. Nelson, Nature Mater. 7, 158 (2008).

${ }^{8}$ L. H. Nguyen, H. Hoppe, T. Erb, S. Günes, G. Gobsch, and N. S. Sariciftci, Adv. Funct. Mater. 17, 1071 (2007).

${ }^{9}$ F. Padinger, R. S. Rittberger, and N. S. Sariciftci, Adv. Funct. Mater. 13, 85 (2003).

${ }^{10}$ D. W. Zhao, P. Liu, X. W. Sun, S. T. Tan, L. Ke, and A. K. K. Kyaw, Appl. Phys. Lett. 95, 153304 (2009).

${ }^{11}$ P. J. Brown, D. S. Thomas, A. Köhler, J. S. Wilson, J.-S. Kim, C. M. Ramsdale, H. Sirringhaus, and R. H. Friend, Phys. Rev. B 67, 064203 (2003)

${ }^{12}$ P. A. Steward, J. Hearn, and M. C. Wilkinson, Adv. Colloid Interface Sci. 86, 195 (2000).

${ }^{13}$ D. P. Sheetz, J. Appl. Polym. Sci. 9, 3759 (1965).

${ }^{14}$ T. Wang, L. Huang, J. Yang, H. Tian, Y. Geng, and D. Yan, J. Phys. Chem. B 114, 16408 (2010). 